\title{
Avoidance of Wrong Level Surgery in the Lumbar Spine: A Technical Report
}

Kingsley R. Chin ${ }^{1}$, Fabio J.R. Pencle ${ }^{2}$, Jonathan Kubik ${ }^{3}$, André V. Coombs ${ }^{4}$, Jason A. Seale ${ }^{2}$, Neel Desai ${ }^{5}$ and Vanessa B Cumming $^{4}$

${ }^{1}$ Charles E. Schmidt College of Medicine at Florida Atlantic University and Less Exposure Surgery Specialists Institute (LESS Institute), Florida, USA

${ }^{2}$ Less Exposure Surgery Specialists Institute (LESS Institute), Florida, USA

${ }^{3}$ Charles E. Schmidt College of Medicine at Florida Atlantic University, Florida, USA

${ }^{4}$ Less Exposure Surgery (LES) Society, Florida, USA

${ }^{5}$ Duke University, Durham, NC 27708, USA

\begin{abstract}
Study Background: Wrong level surgery remains one of the detrimental technical mistakes that can occur during a surgeon's career. There is a higher frequency of wrong site surgery in the lumbar spine compared to the cervical spine. The authors aim to present a localization technique designed to aid in the prevention of wrong level surgery with the use of preoperative and intraoperative localization.
\end{abstract}

Methods: We prospectively followed 240 patients who underwent less exposure surgery for the lumbar spine. We identified the correct operative level using a combination of a 22-guage spinal needle placed under fluoroscopy in the lamina of the vertebra of the target intervertebral disc space prior to incision and then placing a hooked dilator between the spinous processes of the intervertebral space to provide actual confirmation within the operative space.

Results: Data showed that all 240 patients had surgery performed on the correct level using the described technique and the placement of the percutaneous needle assisted in minimizing the incision size to less than $5 \mathrm{~cm}$ for a single level fusion in all cases.

Conclusion: The described technique allows for localization prior to incision, smaller incision size, consistent and accurate localization when performing lumbar spinal surgery.

Keywords: Lumbar spine surgery; Wrong level surgery; Wrong site surgery; Sterile spinal needle

Abbreviations: IDS: Intervertebral Disc Space; FDA: Food and Drug Administration; LSTV: Lumbosacral Transitional Vertebrae

\section{Introduction}

Wrong level spinal surgery is one of the more detrimental surgical mistakes that can be made during a surgeon's career. Wrong level surgery exposes patients to additional procedures and risk, almost certainly damages the doctor-patient relationship, and very often results in legal action [1-4]. Although the incidence is low, with rates reported in the literature ranging from 0.09 to 4.5 per 10,000 surgeries performed, it is estimated that $50 \%$ of spine surgeons will perform one wrong level procedure in their career $[3,5]$. As a result, any additions to the armamentarium of techniques aiming to prevent wrong level surgery are welcome and encouraged. Normal anatomical variations in the spine can increase the chance of wrong level surgery, making proper localization crucially important. In particular, the presence of lumbosacral transitional vertebrae may complicate lumbar spinal procedures by making it difficult to correctly identify the proper level while in the operating theatre [6]. Effective use of intraoperative spine level localization techniques, along with careful review of preoperative imaging, allows the surgeon to correctly identify the proper spinal level even when anatomical variations are present [7-9]. Ebraheim et al., investigated and compared the accuracy of identifying lumbar decompression and fusion levels with the use of direct observation and palpation of the anatomic landmarks, versus intraoperative lateral radiography. The authors studied eighty patients and concluded that direct observation and palpation was not a reliable method to localize lumbar fusion levels with only 95\% accuracy [7]. Many techniques designed to prevent wrong level surgery have already been presented in the literature, as numerous authors have outlined and shared their surgical techniques designed to reduce the chance of wrong level surgery [1,5,9-19]. Additionally, both the North American Spine Societies as well as the Joint Commissions on Accreditation of
Healthcare Organizations have established guidelines to be followed during surgery in order to prevent wrong level procedures [20]. However, a thorough search of the literature revealed no reports that utilized our localization technique of using a spinal needle fixed in the lamina of the vertebra above the target disc space, followed by insertion of a dilator prior to decompression. It has already been demonstrated in a previous study, which this technique proved successful in localization for three patients undergoing spinal surgery in the thoracic spine [21]. Herewith, we present our technique that has been shown to be accurate in correctly identifying the target spinal level in 240 lumbar procedures.

\section{Methods}

We conducted a retrospective analysis of patients who underwent less exposure lumbar spine surgery performed by a single senior surgeon in six different surgical facilities. A total of 240adult patients underwent lumbar spine surgery using this localization technique. IRB approval was obtained for the study for our institution

\section{Technique Description}

The patients were prepared and positioned in the standard manner on the operating table. In order to properly visualize the target spinal

*Corresponding author: Kingsley R. Chin, Charles E. Schmidt College of Medicine at Florida Atlantic University and Less Exposure Surgery Specialists Institute (LESS Institute), 1100 W. Oakland Park Blvd. Suite \#3 Fort Lauderdale, FL, USA, Tel: 954640-6010; Fax: 855-411-4647; E-mail: kingsleychin@gmail.com

Received August 24, 2015; Accepted September 08, 2015; Published September 10, 2015

Citation: Chin KR, Pencle FJR, Kubik J, Coombs AV, Seale JA, et al. (2015) Avoidance of Wrong Level Surgery in the Lumbar Spine: A Technical Report. J Spine 4: 257.doi:10.4172/2165-7939.1000257

Copyright: $\odot 2015$ Chin KR, et al. This is an open-access article distributed under the terms of the Creative Commons Attribution License, which permits unrestricted use, distribution, and reproduction in any medium, provided the original author and source are credited. 




Figure 1: A 22-guage spinal needle subcutaneously placed at the lamina of the superior vertebra of the target intervertebral disc space.



Figure 2: Lateral fluoroscopic view of 22-gauge spinal needle in the lamina of the $L 4$ vertebra.

level, fluoroscopic anteroposterior and lateral views were obtained of the lower lumbar spine with the lumbosacral junction in view. A 22-guage spinal needle was then placed in the lamina of the superior vertebra of the target intervertebral disc space [IDS], followed by fluoroscopic confirmation (Figures 1 and 2). This needle was kept in place after the incision was made in order to maintain our location when the spine was exposed. The target level minimal exposure incision was made and then minimal dissection to the level of the spinous processes. A hooked dilator was placed between the superior and inferior spinous processes of the target IDS, followed by confirmation on fluoroscopy (Figures $3-5)$. Once the target location was confirmed, the specific procedures required for each patient at that spinal level could then proceed.

\section{Results}

Correctly placed instrumentation was confirmed with postoperative imaging for all 240 patients (Table 1). Data obtained from patients showed that all procedures were performed at the correct level using the described technique. Of note, 48 patients (20\%) were found to have lumbosacral transitional vertebrae (LSTV) on preoperative imaging.



Figure 3: A dilator was placed between the superior and inferior spinous processes of the target intervertebral disc space.

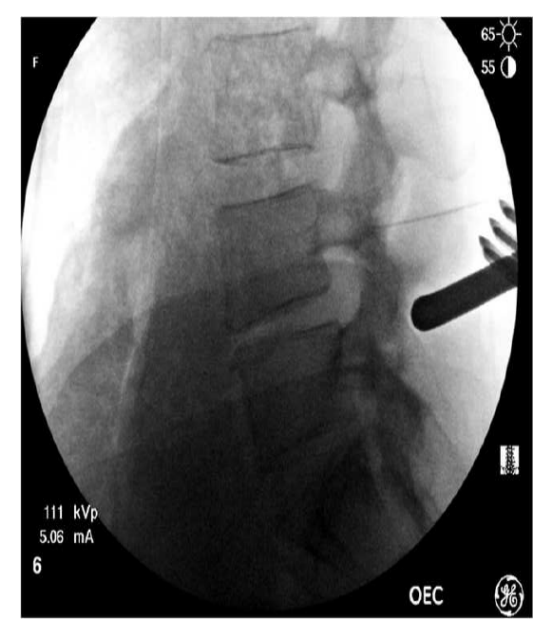

Figure 4: Lateral fluoroscopic confirmation of the dilator placed between the spinous processes of L4 and L5.

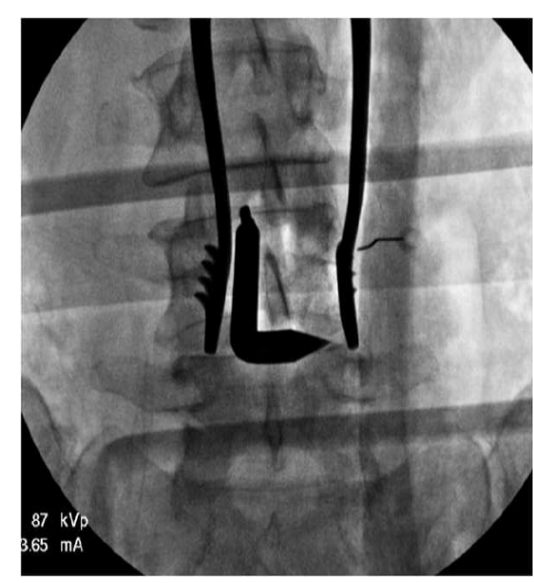

Figure 5: Anteroposterior fluoroscopic image showing 22-gauge spinal needle and dilator in situ. 
Citation: Chin KR, Pencle FJR, Kubik J, Coombs AV, Seale JA, et al. (2015) Avoidance of Wrong Level Surgery in the Lumbar Spine: A Technical Report. J Spine 4: 257.doi:10.4172/2165-7939.1000257

Page 3 of 3

\begin{tabular}{|c|c|c|c|}
\hline Levels & $\mathbf{1}$ & $\mathbf{2}$ & $\mathbf{3}$ \\
\hline No. patients & 156 & 60 & 24 \\
\hline$\%$ patients & $65 \%$ & $25 \%$ & $10 \%$ \\
\hline
\end{tabular}

Levels $=$ number spinal levels operated on for each patient.

Table 1: Breakdown of Procedural Levels $(n=240)$.

\section{Discussion}

The majority of wrong level spine surgery occurs in the lumbar spine with reports has high as $71 \%[3,22]$. Prevention training of residents and fellows is of utmost importance as demonstrated by Mesfin et al. [23]. Although various techniques have previously been described to help to reduce the risk of this occurrence $[9,10]$, it is important to share new, successful techniques aimed toward decreasing the incidence of wrong level spine surgery. Surgeons should develop a standard practice and protocol to prevent wrong level surgery [24]. The aim of this technique was to prevent wrong level spine surgery. It is important to highlight the benefits of this simple technique. Initial identification of operative level prior to incision with confirmation using fluoroscopy. Needle can be left in place or repositioned to confirm level in both open and percutaneous procedures. The 22-gauge spinal needle is readable available in all operating rooms establishing our technique as a convenient option to ensure patient safety without having to purchase additional instruments. In performing spine level localization prior to incision, the size of incision will invariably be smaller and localized to affected level.

One limitation of this technique arises in patients with lumbosacral abnormalities. Due to the limited field of view of intraoperative fluoroscopic images, it is difficult to correctly identify the proper spinal level when these transitional vertebrae are present. The prevalence of these transitional vertebrae has been reported to vary widely, with prevalence rates ranging from $4 \%$ to $35 \%[6,25]$, with the most common variant Castellvi Type 1A [25]. Thorough review of the preoperative imaging helped to identify these abnormalities, as was done with 48 patients found to have LSTV after which, we planned the surgical procedure accordingly. As with all other localization techniques, it is important to use multiple strategies to accurately identify the correct level in spinal surgery. Additional research is necessary to fully assess the use of this technique for spinal surgery on other regions of the spine. Herewith, we presented a technique for localization during lumbar spine surgery procedures to be used addition to standard preoperative protocols. Advantages include a cost effective and easily accessible instrument, potential for reduction in open surgery times, continuous localization and visualization during the procedure, and ultimately reduced risk of patient morbidity. This technique assures the surgeon that lumbar spinal surgery can be consistently performed on the correct spinal level.

\section{Competing Interests and Sources of Funding}

We did not seek or receive any funding from the National Institutes of Health (NIH), Welcome Trust, Howard Hughes Medical Institute (HHMI), or others for this work. KRC is a shareholder in and receives other benefits from Spine Frontier Inc., neither of the other authors (FJRP, JK, AVC, JAS, ND and VBC) have any potential conflicts of interest to declare for this work.

\section{References}

1. Nowitzke A, Wood M, Cooney K (2008) Improving accuracy and reducing errors in spinal surgery--a new technique for thoracolumbar-level localization using computer-assisted image guidance. The spine journal : official journal of the North American Spine Society 8(4): 597-604.

2. Fager CA (2006) Malpractice issues in neurological surgery. Surg Neurol 65: 416-421.

3. Mody MG, Nourbakhsh A, Stahl DL, Gibbs M, Alfawareh M, et al. (2008) The prevalence of wrong level surgery among spine surgeons. Spine 33: 194-198.

4. Goodkin R, Laska LL (2004) Wrong disc space level surgery: medicolegal implications. Surgical neurology 61: 323-341.

5. Devine J, Chutkan N, Norvell DC, Dettori JR (2010) Avoiding wrong site surgery: a systematic review. Spine (Phila Pa 1976) 35: S28-36.

6. Konin GP, Walz DM (2010) Lumbosacral transitional vertebrae: classification, imaging findings, and clinical relevance. American journal of neuroradiology 31: $1778-1786$.

7. Ebraheim NA, Inzerillo C, Xu R (1999) Are anatomic landmarks reliable in determination of fusion level in posterolateral lumbar fusion? Spine (Phila $\mathrm{Pa}$ 1976) 24(10): 973-974

8. Ammerman JM, Ammerman MD (2008) Wrong-sided surgery. J Neurosurg Spine 9(1): 105-106

9. Ammerman JM, Ammerman MD, Dambrosia J, Ammerman BJ (2006) A prospective evaluation of the role for intraoperative $\mathrm{x}$-ray in lumbar discectomy. Predictors of incorrect level exposure. Surg Neurol 66(5): 470-473.

10. Binning MJ. Schmidt MH (2010) Percutaneous placement of radiopaque markers at the pedicle of interest for preoperative localization of thoracic spine level. Spine 35: 1821-1825.

11. Bolger C, Wigfield C (2000) Image-guided surgery: applications to the cervical and thoracic spine and a review of the first 120 procedures. Journal of neurosurgery 92: 175-180.

12. Henegar MM, Vollmer DG, Silbergeld DL (1996) Intraoperative transligamentous ultrasound in the evaluation of thoracic intraspinal disease. Technique Spine 21(1): 124-127.

13. Hsu W, Sciubba DM, Sasson AD, Khavkin Y, Wolinsky JP, et al. (2008) Intraoperative localization of thoracic spine level with preoperative percutaneous placement of intravertebral polymethylmethacrylate. Journal of spinal disorders \& techniques. 21: 72-75.

14. Irace C, Corona C (2010) How to avoid wrong-level and wrong-side errors in lumbar microdiscectomy. Journal of neurosurgery. Spine 12(6): 660-665.

15. Redfern RM, Smith ET (1986) A method for identification of vertebral level. Annals of the Royal College of Surgeons of England 68: 163.

16. Rosahl SK, Gharabaghi A, Liebig T, Feste CD, Tatagiba M, et al. (2002) Skin markers for surgical planning for intradural lesions of the thoracic spine. Technical note. Surgical neurology 58: 346-348.

17. Singh H, Scott MA, Hecht AC, Jenkins AL (2009) Novel fluoroscopic technique for localization at cervicothoracic levels. Journal of spinal disorders \& techniques 22: 615-618.

18. Upadhyaya CD, Wu JC, Chin CT, Balamurali G, Mummaneni PV (2012) Avoidance of wrong-level thoracic spine surgery: intraoperative localization with preoperative percutaneous fiducial screw placement. Journal of neurosurgery. Spine 16: 280-284.

19. Paolini S, Ciappetta P, Missori P, Raco A, Delfini R (2005) Spinous process marking: a reliable method for preoperative surface localization of intradura lesions of the high thoracic spine. British Journal of Neurosurgery 19: 74-76.

20. Organizations JCotAoH.(2013) 20 SRC

21. Chin KR, Seale J, Cumming V (2013) Avoidance of Wrong-Level Thoracic Spine Surgery Using Sterile Spinal Needles. J Spinal Disord Tech.

22. Jhawar BS, Mitsis D, Duggal N (2007) Wrong-sided and wrong-level neurosurgery: a national survey. J Neurosurg Spine 7: 467-472.

23. Mesfin A, Canham C, Okafor $L$ (2015) Prevention training of wrong-site spine surgery. J Surg Educ 72: 680-684.

24. Hsiang J (2011) Wrong-level surgery: A unique problem in spine surgery. Surg Neurol Int 2: 47

25. Apazidis A, Ricart PA, Diefenbach CM, Spivak JM (2011) The prevalence of transitional vertebrae in the lumbar spine. Spine J 11(9): 858-862. 